\title{
Déroulement des premiers instants suivant l'explosion d'une bombe historique posée sur le fond marin
}

\section{Thierry GARLAN ${ }^{1}$, Xavier DEMOULIN ${ }^{2}$, Michel ARRIGONI ${ }^{3}$, Nathalie FAVRETTO-CRISTINI ${ }^{4}$, Éric BEUCLER ${ }^{5}$, Anne DESCHAMPS ${ }^{6}$}

1. SHOM, Département Géologie Marine, 13, rue du Chatellier - CS 92803 - 29228 Brest Cedex 2, France. thierry.garlan@shom.fr

2. MAREE, 15 rue Galilée, 56270 Ploemeur, France. xdemoulin@maree.fr

3. ENSTA Bretagne, Institut de Recherche Dupuy de Lôme - CNRS UMR 6027, 2, rue François Verny - 29806 Brest Cedex 9, France. michel.arrigoni@ensta-bretagne.fr

4. Aix-Marseille Univ, CNRS, Centrale Marseille, LMA, Marseille, France, 4 impasse Nikola Tesla, CS 40006, F-13453 Marseille Cedex 13, France. favretto@lma.cnrs-mrs.fr

5. Université de Nantes, UFR Sciences et Techniques, UMR 6112 CNRS - LPG, Bâtiment 4, 2 rue de la Houssinière, BP 92208, 44322 Nantes Cedex 3, France. eric.beucler@univ-nantes.fr

6. Université Côte d'Azur, CNRS, Observatoire de la Côte d'Azur, IRD, Géoazur, Bâtiment 3, 250 rue Albert Einstein, 06560 Sophia Antipolis, France.

\section{Résumé :}

Lors de l'explosion de bombes historiques en domaine marin, des séismes peuvent être ressentis par les riverains. Afin de limiter ces risques, l'ANR POSA (caractérisation de la Propagation des Ondes Sismiques d'origine Anthropique), conduite de 2014 à 2019, concerne l'étude de la relation entre le poids de la charge explosive, les caractéristiques du fond marin, et la propagation sismique engendrée, à partir de deux expérimentations durant lesquelles 16 explosions d'une charge totale de 2,6 t équivalent TNT, ont été réalisées par les Plongeurs Démineurs de la Marine. L'analyse des expérimentations a permis de caractériser les ondes issues de ces explosions et de les modéliser afin de prédire à terme l'intensité des séismes créés par ces explosions.

Mots-clés :

Génie côtier, Explosion sous-marine, Propagation, Microséisme, Epaisseur de Sédiments, Modélisation sismo-acoustique 


\section{Thème 7 - Risques côtiers}

\section{Introduction}

La destruction d'engins explosifs historiques est une activité continue dans le monde ; et la découverte chaque semaine, sur le territoire français, de bombes de plusieurs centaines de kilogramme équivalent TNT, provenant de la seconde et même de la première guerre mondiale, montre que ce problème perdurera en environnement marin durant plusieurs décennies voire plusieurs siècles. Les opérations de déminages ont débuté dès la fin de la seconde guerre mondiale et la plupart des études sur les processus mis en jeu ont eu lieu durant les 20 années suivantes. L'ANR POSA a été construite comme une mise à jour de ces données anciennes avec des moyens modernes comprenant par une approche pluridisciplinaire la mesure de la propagation des ondes dans des environnements bien caractérisés puis la simulation de ces environnements et explosions pour évaluer la capacité à reproduire les séismes ressentis. Des résultats inattendus ont engendré des études initialement non prévues sur les traces des impacts des explosions au sol et en surface, sur le calcul de la localisation et du temps T0 des explosions et sur les modes de propagation et d'évolution au cours du trajet des ondes. Enfin une étude des différences de la propagation lors de l'explosion des engins sur le fond ou en en pleine eau a été réalisée. Etant donné la multiplicité des domaines scientifiques abordés, plusieurs articles sont en cours de rédaction celui-ci concerne plus spécifiquement le suivi de l'onde propagée en champ proche. Lors du contre-minage d'engins explosifs historiques en domaine marin, des séismes sont parfois ressentis par les riverains et peuvent aller jusqu'au bris de vitres pour des environnements géologiques particuliers. Afin de limiter ce risque, le projet POSA financé par l'ANR et la DGA, s'est déroulé de 2015 à 2019 dans le cadre d'une collaboration entre le Shom, les Universités de Marseille, Nice et Nantes, la société MAREE, l'ENTSA-Bretagne et plusieurs services de la défense chargés des opérations de déminage en Méditerranée. Le centre du sujet était l'étude de la relation entre le poids de la charge explosive, les caractéristiques du fond marin, et la propagation sismique engendrée, à partir de deux expérimentations durant lesquelles 16 explosions d'une charge totale de 2,6 t équivalent TNT, ont été réalisées par les plongeurs démineurs de la Marine en Grande Rade de Toulon et dans la Rade d'Hyères. L'analyse des données enregistrées par les stations sismologiques et les hydrophones déployés le long du rivage et en mer, ont permis de caractériser les spécificités des ondes produites par ces explosions. Sur la base de ces mesures et de modèles 3D des caractéristiques physiques et géologiques du sol et du sous-sol, des simulations sismo-acoustique ont été réalisées afin d'évaluer la possibilité de prédire l'intensité des séismes créés par ces explosions.

Les résultats du projet reposent sur deux expérimentations réalisées sur deux secteurs préalablement étudiés par des levés géologiques. Avec l'explosion de 8 charges de 5 à $120 \mathrm{~kg}$ équivalent TNT, la première expérimentation de 2016 en Grande-Rade de Toulon, a fourni de premiers résultats sismiques, permis de premières modélisations et défini les charges et l'instrumentation devant être mises en œuvre lors de la seconde expérimentation. Cette dernière s'est déroulée en décembre 2018 en Rade d'Hyères. Avec 


\section{XVI'̀mes Journées Nationales Génie Côtier - Génie Civil \\ Le Havre 2020}

un vaste spectre d'explosions, allant de 80 à $680 \mathrm{~kg}$ éq. TNT, offrant de très nombreuses données sismologiques et acoustiques d'excellente qualité et permettant la réalisation de modélisations très haute résolution au plus proche des conditions réelles.

\section{Présentation de l'ANR POSA}

Chaque mois la marine doit gérer la destruction de dizaines d'engins explosifs historiques. Bien que souvent enregistrés par les réseaux sismologiques permanents, les signaux émis par ces explosions sur le fond marin ou en sub-surface sont très peu étudiés. D'une part car les heures exactes des explosions ne sont pas connues et d'autre part, car compte tenu du faible nombre de stations à proximité des côtes, les incertitudes en termes de localisation et de détermination de temps origine sont grandes. L'ANR POSA concerne la question de gestion des risques en amont des opérations de contre-minage en domaine marin. Pour cela il a caractérisé la relation charge explosive/fond marin/propagation des ondes à partir de l'étude de mesures sismologiques et acoustiques, lors de déminages d'engins explosifs de diverses puissances dans deux environnements puis de la reproduction des phénomènes par modélisation. Ces travaux reposent sur :

a) la réalisation d'un modèle géologique $3 \mathrm{D}$ des zones d'expérimentation,

b) des expérimentations durant lesquelles a été mesurée la propagation de l'onde sismique au moyen de capteurs en mer et de sismomètres à terre à proximité du rivage,

c) le développement et l'utilisation d'outils de simulation sismo-acoustique réalistes, c'est-à-dire le plus proche possible du contexte, afin de comparer les résultats de modélisation aux mesures effectuées lors des opérations de contre-minages.

\section{Impact lors des premières millisecondes suivant l'explosion}

Lorsqu'un contre-minage est réalisé, l'explosion engendre deux phénomènes immédiats :

a) un disque à la surface de la mer qui apparaît instantanément et qui est suivi quelques secondes plus tard par l'arrivée de la bulle de cavitation qui en surface forme un bombement ou une gerbe selon la charge et la profondeur,

b) une empreinte circulaire sur le fond qui n'avait encore jamais été décrite (GARLAN et al., 2018).

\subsection{Empreinte de surface}

Certains engins explosifs étant des cylindres, afin de déterminer si la propagation engendrée était isotrope une étude de l'empreinte de surface a été réalisée pour voir si elle était un cercle ou une ellipse. Des photographies et vidéos ont été prises lors des explosions à partir des bateaux de la Marine et depuis la terre, mais aucune prise de vue aérienne n'ayant pu être réalisée, cette question reste posée.

Dans les millisecondes suivant l'explosion d'un engin posé sur le fond, l'onde de choc engendre en surface un disque blanc qui est inclus dans un second disque défini par un changement de la rugosité de surface (voir figure 1). 


\section{Thème 7 - Risques côtiers}

Les mesures acoustiques et de la sonde de choc (Shock-gauge-transducer T11, www.neptune-sonar.co.uk) montrent que ce phénomène se passe dans les $15 \mathrm{~ms}$ suivant l'explosion. Une mesure de ces disques, prenant comme référence la taille des bateaux et donc demeurant assez imprécise, donne pour dimension du diamètre du disque blanc de surface : $0,19 \mathrm{x}$ poids de la charge en équivalent TNT +78 (voir figure 2). Le diamètre du disque de plus grande dimension, caractérisé par une forte rugosité de surface, est deux fois plus large. Ainsi, pour une bombe d'une tonne équivalent TNT, le rayon des deux cercles est respectivement de 130 et $260 \mathrm{~m}$. Il semblerait ainsi possible de définir la charge à partir de la dimension des disques de surface, mais il serait nécessaire pour cela de réaliser de telles mesures pour différentes profondeurs et différents environnements.

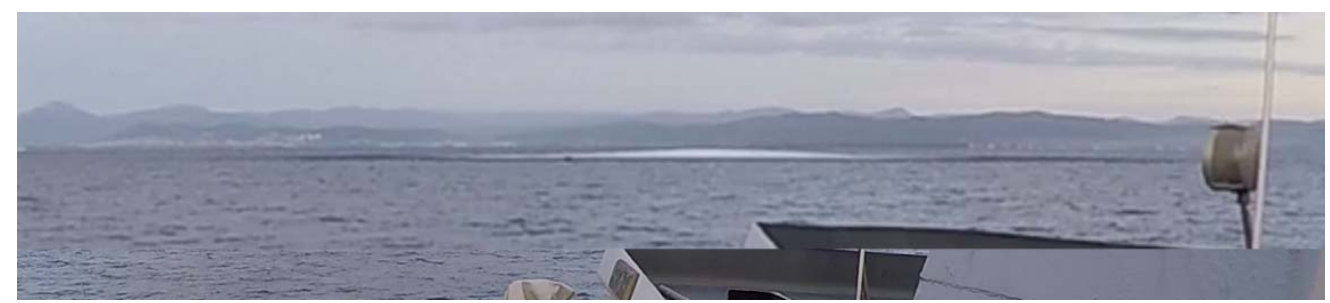

Figure 1. Disques de surface provoqués par l'onde de choc vue d'un bâtiment de la marine lors de l'expérimentation de décembre 2018 en Rade d'Hyères.

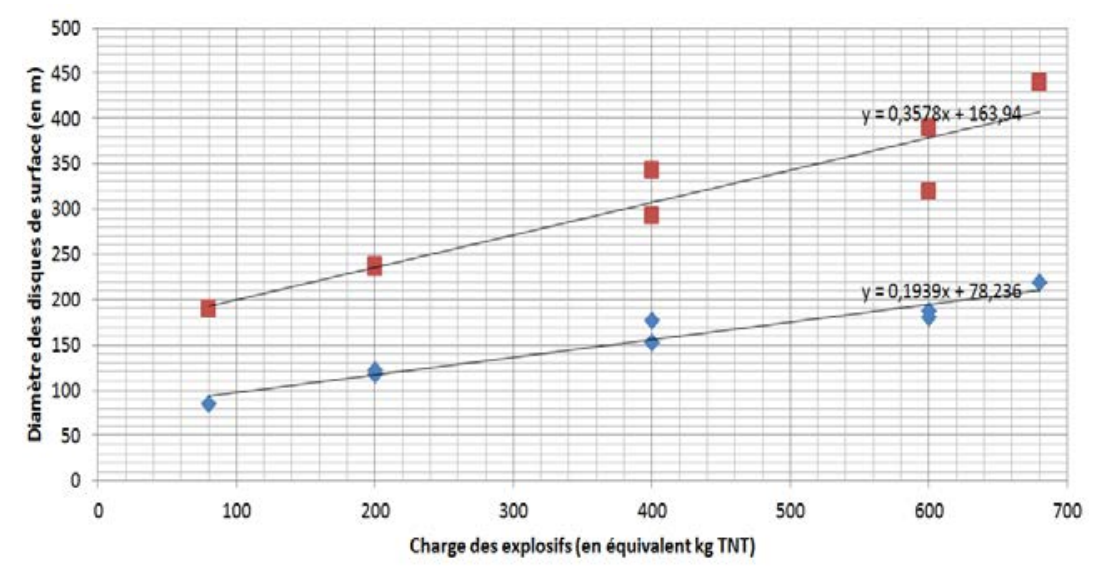

Figure 2. Diamètres des deux cercles de surface provoqués par l'onde de choc en fonction de la charge des explosifs.

\subsection{Cavitation}

Trois enregistreurs mis en œuvre par la société MAREE en collaboration avec l'ENSTA Bretagne ont permis la collecte de données à proximité de la zone de cavitation. Une sonde de choc T11, apte à mesurer des pressions jusqu'à $275 \mathrm{MPa}$ avec une fréquence d'échantillonnage de $625 \mathrm{kHz}$ a été déployée à environ $100 \mathrm{~m}$ des sites de tirs pour estimer les niveaux et la forme d'onde émise, et deux hydrophones placés à plus grande distance, choisis pour ne pas saturer, ont enregistré les signaux propagés dans l'eau à la fréquence 


\section{XVIèmes Journées Nationales Génie Côtier - Génie Civil \\ Le Havre 2020}

d'échantillonnage de $78 \mathrm{kHz}$. Ce jeu de données permet de décrire la phénoménologie des signatures des ondes mécaniques émises lors des explosions et d'analyser leur propagation. Ces données montrent l'impact de l'onde de choc directe, vers $160 \mathrm{~ms}$, puis réfléchie par la surface, vers $162 \mathrm{~ms}$ (voir figure 3). L'onde de choc est la plus importante dans l'eau. Plus la charge est importante et proche du capteur et plus la fréquence de l'onde de choc augmente $(150-250 \mathrm{~Hz})$. Pour la charge de $400 \mathrm{~kg}$ éq. TNT constitué de deux charges de $200 \mathrm{~kg}$ attachées ensembles, s'observe un écart d'une milliseconde entre l'explosion des deux charges, ce qui correspondrait soit à un retard pyrotechnique d'une milliseconde, soit à l'écart de près d'un mètre entre le centre des deux explosifs démontrant ainsi l'ajout d'une complexité lors d'explosions d'explosifs composites.
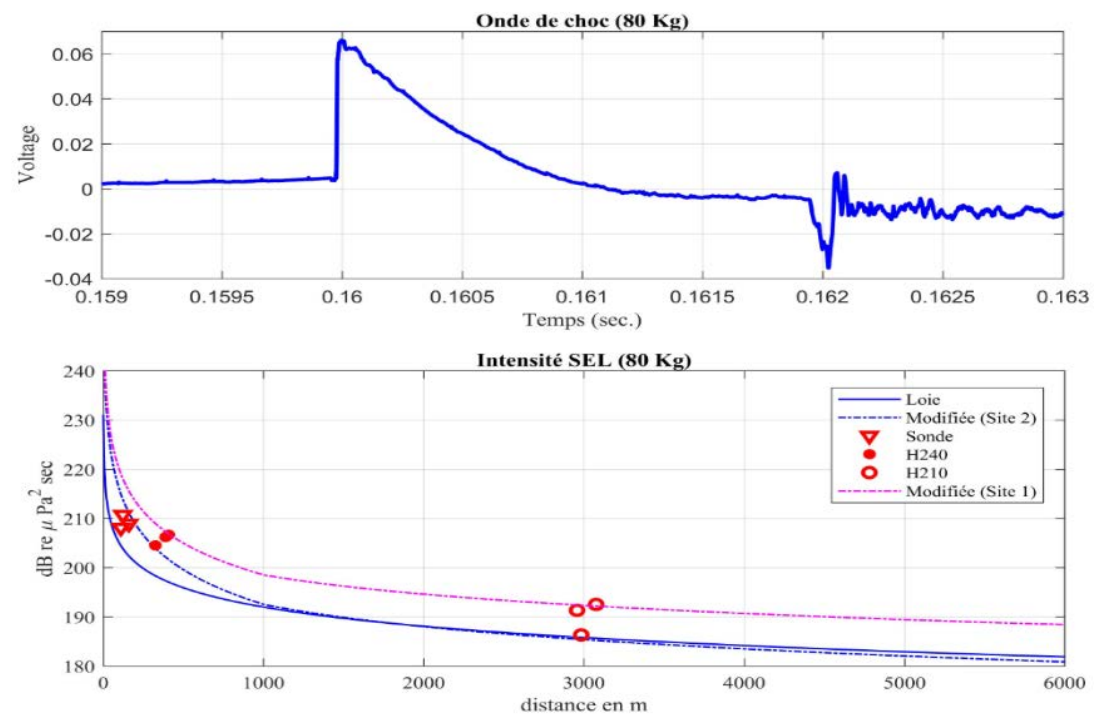

Figure 3. Haut : signal temporel de l'onde de choc primaire pour une charge de $80 \mathrm{~kg}$ posée sur le fond. Bas: Calcul de SEL (Sound Exposure Level de SOLOWAY \& DAHL, 2014) avec la loi antérieure à l'expérimentation POSA (-) et modifiée suite à l'expérimentation pour les deux sites d'explosion (---- ----).

Une modélisation numérique de la propagation du signal d'explosion en champ proche, à une distance de $200 \mathrm{~m}$, à l'aide d'un code hydrodynamique eulérien SPEED pour le cas type d'une explosion de $80 \mathrm{Kg}$, par $30 \mathrm{~m}$ de fond montre d'une part l'évolution du signal dans les premiers stades de sa propagation, et d'autre part, les premières étapes de de la formation d'un cratère dans le fond. Ces observations soulignent l'importance de réaliser des mesures aussi proches de la source que possible afin de mieux définir, dans les modélisations, les caractéristiques de l'émission.

\subsection{Empreinte sur le fond}

Des disques de forte réflectivité sont également observables sur le fond, sur les mosaïques issues des sondeurs multifaisceaux et des sonars latéraux. Il s'agit là de cercles, et ceux- 


\section{Thème 7 - Risques côtiers}

ci sont pérennes et permettent donc une étude plus aisée. Contrairement à ce que l'on pourrait attendre il ne s'agit pas de cratères, mais de zones plates, qui présentent parfois une petite dépression centrale de quelques mètres de diamètre. Leur analyse montre qu'ils ne correspondent à aucune structure sédimentaire connue (GARLAN et al., 2018). Près de 700 cercles appelés blast marks, ont été observés sur la Grande-Rade de Toulon soit 30 blast marks $/ \mathrm{km}^{2}$. Les plus grands atteignent $134 \mathrm{~m}$, mais seuls $2 \%$ d'entre eux dépassent 100 mètres de diamètre, les diamètres étant le plus souvent compris entre 20 et 50 mètres. Ces cercles tendent à être plus nets vers les profondeurs les plus importantes, qui peuvent dépasser $200 \mathrm{~m}$, et ne sont pas observés par petits fonds dans la zone $0-15 \mathrm{~m}$ (voir figure 4). Ceci semble provenir d'une remobilisation sédimentaire par les vagues, qui dans cette région sont actives jusqu'à des profondeurs de 9 à $10 \mathrm{~m}$, et qui effectueraient un gommage de ces traces. Ces petits fonds sont le lieu des herbiers de posidonie qui masquent probablement ces marques ainsi que les engins explosifs historiques. Ces blast-marks sont beaucoup trop nombreux pour correspondre aux opérations de déminages et seuls quelques-uns d'entre eux pourraient en provenir. Il est ainsi probable qu'ils proviennent des bombardements effectués durant la seconde guerre mondiale durant l'opération Dragon d'août 1944 et des bombardements les ayant précédés.

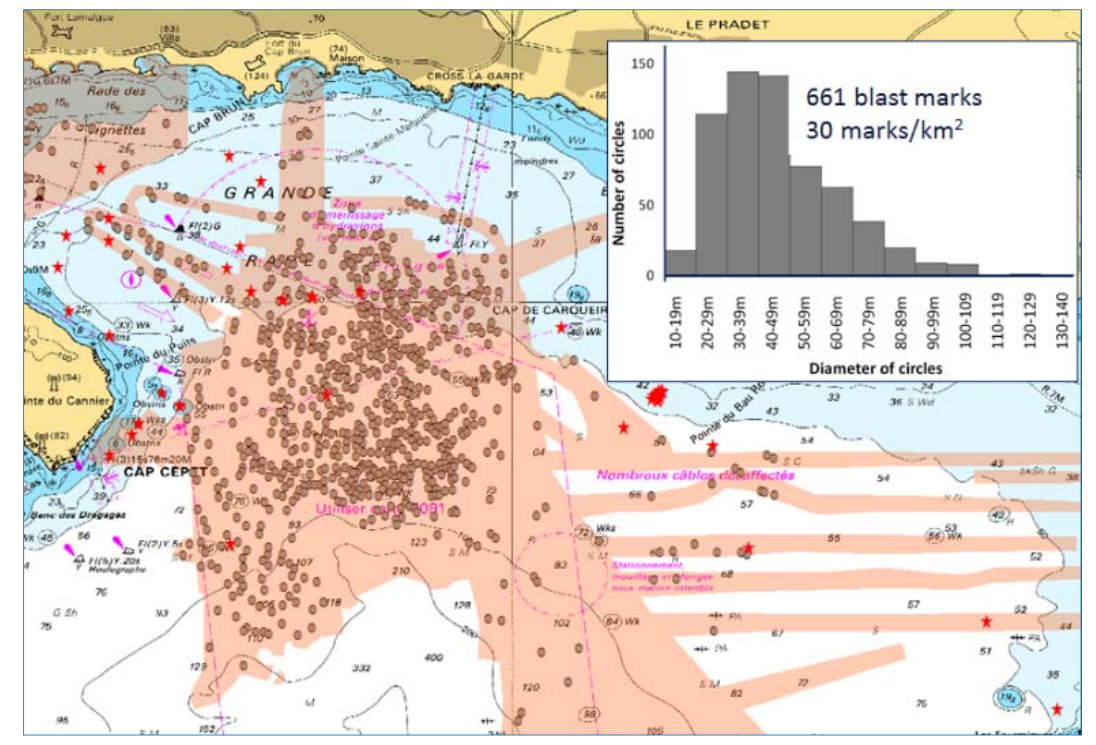

Figure 4. Localisation des blast marks : empreintes circulaires détectées par SMF

(Sondeur MultiFaisceaux) sur le fond de la Grande-Rade de Toulon, et position des contre-minages effectués depuis 2010 : *.

\section{Développement de la bulle de gaz et la propagation dans l'eau}

Après l'onde de choc se développe la première oscillation de la bulle de gaz qui engendre le signal de l'onde de choc secondaire (voir figure 5). Elle se caractérise par de plus basses fréquences, de 20 à $30 \mathrm{~Hz}$, qui à l'opposé de l'onde de choc se propagent plutôt par le sol. 


\section{XVIèmes Journées Nationales Génie Côtier - Génie Civil \\ Le Havre 2020}

Ces oscillations de la bulle de gaz pourraient être à l'origine des séismes ressentis à terre. La différence entre l'intensité près de la source et l'intensité mesurée à plusieurs kms fournit les pertes de propagation dans l'eau. Il a ainsi été possible de calculer un paramètre caractérisant distinctement la propagation pour les 2 sites de la Rade d'Hyères. Celle-ci peut être modélisée par la forme d'onde analytique de Friedlander. Deux lois empiriques sont classiquement utilisées pour prédire l'intensité Peak du signal de pression d'une part, et l'énergie du signal d'autre part, en fonction de la charge à la puissance $1 / 3$ et en fonction de la distance. L'expérimentation POSA a permis d'affiner ces lois empiriques qui ne prennent pas en compte l'impact de l'environnement. La loi empirique SEL (voir figure 3) appliquée à trois explosions de charges de $80 \mathrm{~kg}$ montre des résultats systématiquement au-dessus des résultats proposés par les lois empiriques. Ceci souligne l'importance de l'effet de site sur les résultats. Ceci met en évidence le besoin d'étendre de telles mesures sur des environnements géologiquement différents.

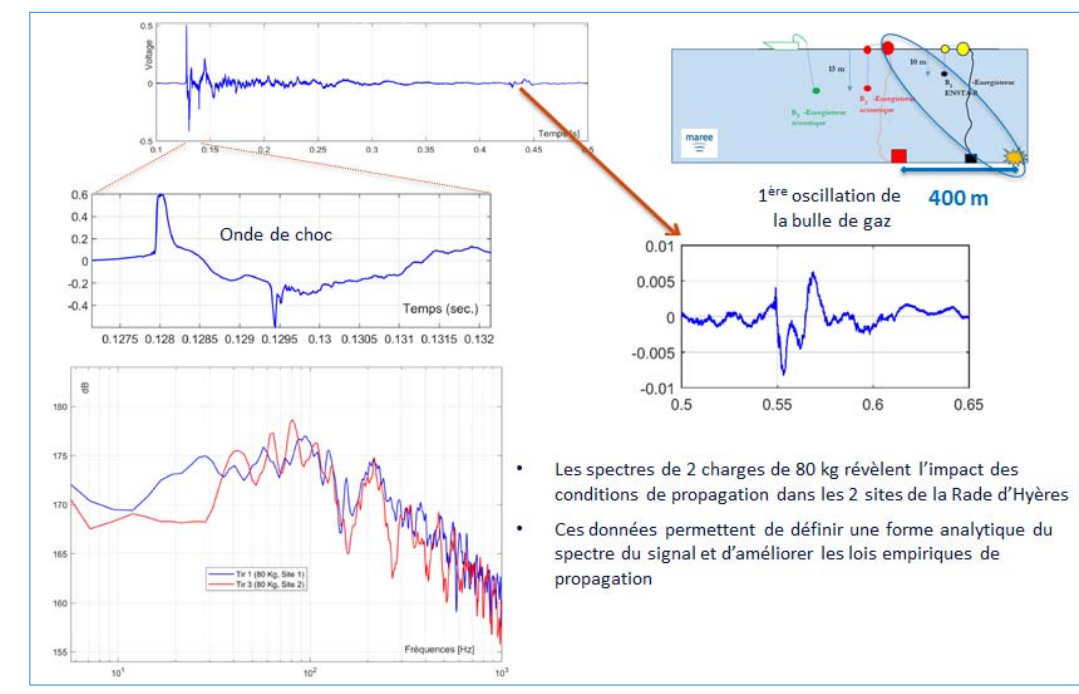

Figure 5. Oscillation de la bulle de gaz perçue à 400m de l'explosion après 55 ms et comparaison de 2 spectres de mêmes charges sur 2 sites différents.

La synthèse des empreintes, fugaces en surface de l'eau et pérennes sur le fond, sont résumées sur un modèle théorique (voir figure 6). En partant du principe que les plus gros blast-marks proviennent d'engins d'une tonne éq. TNT (charge classique des bombes les plus importantes) ce schéma conceptuel donne un doublement des diamètres entre le fond et le disque blanc de surface et entre celui-ci et la zone de surface perturbée. Ces résultats doivent être confirmés par d'autres mesures et/ou par modélisation car ils pourraient varier avec la profondeur et la géologie (épaisseur sédimentaire, proximité du socle rocheux, ...). 


\section{Thème 7 - Risques côtiers}

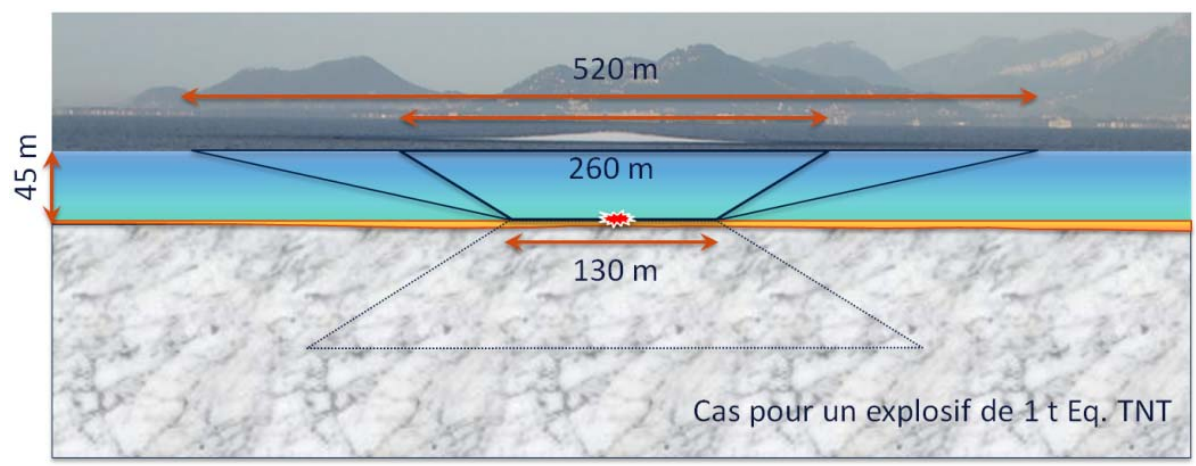

Figure 6. Schéma conceptuel des empreintes de surface et sur le fond lors de l'explosion d'une bombe d'une tonne équivalent TNT.

\section{Conclusions}

L'ANR POSA partait de connaissances bibliographiques anciennes, faute d'études récentes, sur l'émission d'ondes émises par une explosion d'un engin explosif posé sur le fond et sur l'existence de séismes provenant de leur propagation par la roche. Le couplage d'études de géologie (modèle 3D des caractéristiques du sous-sol), de sismologie (17 sismomètres répartis sur le pourtour de la Rade d'Hyères au centre de laquelle ont été contre-minées 8 charges de 80 à $680 \mathrm{~kg}$ éq. TNT), d'acoustique (hydrophones et sonde de choc), a permis la réalisation de modélisations très haute résolution au plus proche des conditions réelles (WANG et al., 2018). L'adaptation des capteurs sismologiques pour obtenir la gamme de fréquence la plus complète possible, l'utilisation de sondes de choc et d'un système de mesure de la célérité dans les sédiments pour atteindre le modèle géologique 3D le plus précis possible, et l'adaptation des modèles ont permis d'atteindre les objectifs du projet, mais aussi de mesurer la complexité de l'onde propagée par ces explosions. Les expérimentations POSA ont dimensionné les effets de l'explosion dans les 15 premières millisecondes avec l'empreinte de l'onde de choc à la surface et sur le fond permettant d'améliorer l'ancien modèle analytique. Elles ont mis en évidence le contenu fréquentiel de l'onde arrivant à terre, et l'existence d'une transmission de ce signal par la roche, le sédiment et la colonne d'eau. Les modélisations basées sur ces mesures soulignent la grande complexité de la propagation, l'impact des poches sédimentaires et la grande importance de la célérité des ondes $\mathrm{S}$ et de leur atténuation dans les sédiments. Ces résultats permettent d'ores et déjà de relativiser l'importance des risques dans des environnements géologiques non fracturés ; car ceux-ci semblent ne pas pouvoir aller audelà du bris de verre et ceci ne peut être atteint que dans des conditions exceptionnelles. Mais les écarts entre modélisation et observation soulignent le besoin de mieux connaître l'émission et sa directivité pour pouvoir quantifier les effets des systèmes de protection tels que les rideaux de bulles et quantifier les effets sur les habitats. L'impact de l'environnement sur des variations des fréquences des ondes propagées et en particulier 


\section{XVIèmes Journées Nationales Génie Côtier - Génie Civil \\ Le Havre 2020}

des phénomènes d'apparition de basses fréquences souligne la nécessité de réaliser de tels travaux dans d'autres environnements géologiques.

\section{Références bibliographiques}

GARLAN T., MATHIAS X., BRENON E., FAVRETTO-CRISTINI N., DESCHAMPS A., BEUCLER E., GUYOMARD P., MORIO O. (2018). Circular Sedimentary Figures of Anthropic Origin in a Sediment Stability Context, Proceedings from the International Coastal Symposium (ICS) 2018, Busan, Republic of Korea, Journal of Coastal Research, Vol. 85 (sp1), pp 411-415. https://doi.org/10.2112/SI85-083.1

SOLOWAY A. G., DAHL P.H. (2014). Peak sound pressure and sound exposure level from underwater explosions in shallow water. Journal of the Acoustical Society of America 136-3, pp 218-223. https://doi.org/10.1121/1.4892668

WANG F., FAVRETTO-CRISTINI N., CRISTINI P., MORIO O., GARLAN T., MATHIAS X., KOMATITSCH D., MONTEILLER V., DESCHAMPS A., BEUCLER E., AMBROIS D. (2018). Numerical modeling of seismo-acoustic wave propagation in the Grande Rade of Toulon. $14{ }^{\text {ème }}$ Congrès Français d'Acoustique, Le Havre 23-27 Avril 2018, pp 335-336. 
Thème 7 - Risques côtiers 